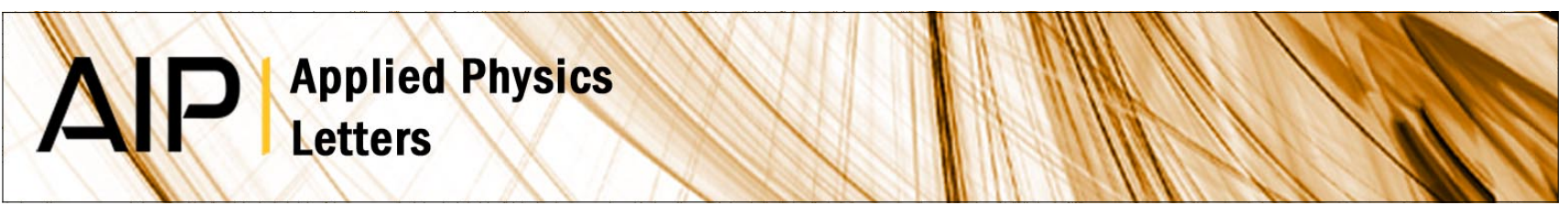

\title{
Capillary origami and superhydrophobic membrane surfaces
}

N. R. Geraldi, F. F. Ouali, R. H. Morris, G. McHale, and M. I. Newton

Citation: Appl. Phys. Lett. 102, 214104 (2013); doi: 10.1063/1.4808015

View online: http://dx.doi.org/10.1063/1.4808015

View Table of Contents: http://apl.aip.org/resource/1/APPLAB/v102/i21

Published by the American Institute of Physics.

Additional information on Appl. Phys. Lett.

Journal Homepage: http://apl.aip.org/

Journal Information: http://apl.aip.org/about/about_the_journal

Top downloads: http://apl.aip.org/features/most_downloaded

Information for Authors: http://apl.aip.org/authors

\section{ADVERTISEMENT}

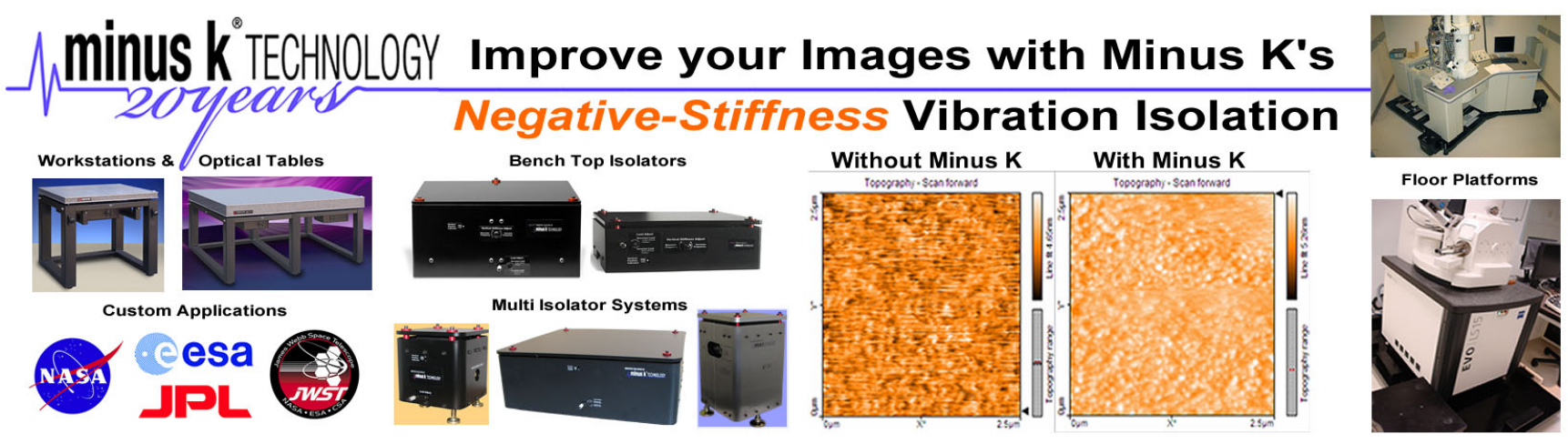




\title{
Capillary origami and superhydrophobic membrane surfaces
}

\author{
N. R. Geraldi, ${ }^{1}$ F. F. Ouali, ${ }^{1, a)}$ R. H. Morris, ${ }^{1}$ G. McHale, ${ }^{2}$ and M. I. Newton ${ }^{1}$ \\ ${ }^{1}$ School of Science and Technology, Nottingham Trent University, Clifton Lane, Nottingham NG11 8NS, \\ United Kingdom \\ ${ }^{2}$ Faculty of Engineering \& Environment, Northumbria University, Ellison Place, \\ Newcastle upon Tyne NE1 8ST, United Kingdom
}

(Received 2 May 2013; accepted 14 May 2013; published online 31 May 2013)

Capillary origami uses surface tension to fold and shape solid films and membranes into three-dimensional structures. It uses the fact that solid surfaces, no matter how hydrophobic, will tend to adhere to and wrap around the surface of a liquid. In this work, we report that a superhydrophobic coating can be created, which can completely suppress wrapping as a contacting water droplet evaporates. We also show that using a wetting azeotropic solution of allyl alcohol, which penetrates the surface features, can enhance liquid adhesion and create more powerful Capillary Origami. These findings create the possibility of selectively shaping membrane substrates. (c) 2013 AIP Publishing LLC. [http://dx.doi.org/10.1063/1.4808015]

The initial development of microsystems technology suffered from the problem that liquid photoresist would often result in capillary bridges between microscale surface features that caused them to deform and clump together. Eventually, this effect was put to positive use to assemble threedimensional shapes from planar (two-dimensional) fabricated structures by rotating surface features using molten solder. ${ }^{1-4}$ The same effect causes the hairs on a wet brush to clump together. ${ }^{5}$ An extension of these ideas has been to use contact with a liquid, typically an evaporating droplet, to completely fold up a membrane substrate into a pre-determined threedimensional shape-an effect called Capillary Origami.$^{6-8}$ This liquid adhesive effect depends on the ratio of the elastic forces maintaining the rigidity of the substrate to the surface tension forces of the liquid trying to shape the substrate. It is now well understood that the controlling parameter for these processes is the elastocapillary length, $L_{\mathrm{EC}}=\left(\kappa_{B} / \gamma_{\mathrm{LV}}\right)^{1 / 2}$, where $\kappa_{B}$ is the bending rigidity of the substrate and $\gamma_{\mathrm{LV}}$ is the surface tension of the liquid, and this sets the critical length scale at which substrates of a given thickness can be shaped by a liquid. Applications of this include the fabrication of microprisms, ${ }^{9}$ iridophores, ${ }^{10}$ and assembling carbon nanotubes $^{11}$ (see also the review by Roman and Bico ${ }^{12}$ ).

A potentially surprising aspect of Capillary Origami is that the liquid adhesion is not suppressed by substrate hydrophobicity. As demonstrated by Gao and McCarthy, ${ }^{13}$ even what is normally regarded as hydrophobic Teflon ${ }^{\circ}$, when made into a thin membrane, will spontaneously wrap itself around a water droplet. This can be explained by considering the surface free energies of an initial non-wrapped and a final wrapped state. ${ }^{14}$ For a smooth membrane substrate with no bending energy, the final wrapped state is always the lower energy state provided the Young's law contact angle is less than $180^{\circ}$. However, this situation is predicted to change when the surface is rough and this introduces a dependence on the contact angle into the problem. ${ }^{14,15}$ In one limit, liquid can be assumed to not penetrate into the surface structure

\footnotetext{
${ }^{\text {a) }}$ Author to whom correspondence should be addressed. Electronic mail fouzia.ouali@ntu.ac.uk
}

(a Cassie-Baxter type state) and this allows a non-adhesive state to be achieved. In the other limit, liquid can be assumed to penetrate into the surface structure (a Wenzel state) enhancing the liquid adhesion so that substrates of greater stiffness can be deformed. A key challenge in suppressing liquid adhesion occurring in Capillary Origami is to produce a completely non-wetting (superhydrophobic) surface coating that does not substantially increase the substrate rigidity.

In this report, we consider the capillary-induced wrapping of triangular shaped films of polydimethylsiloxane (PDMS) possessing a thin surface coating. We use evaporation of a deposited liquid droplet to determine the critical side length at which wrapping occurs. We follow the method of Py et al. ${ }^{6}$ by examining a sequence of equilateral triangular shaped membranes with decreasing side lengths to determine the critical side length, $L_{\text {crit }}$, for which an evaporating droplet can no longer completely fold up the membrane around itself; Py et al. argued that the critical length should be proportional to the elastocapillary length, i.e., $L_{\text {crit }} \propto L_{\mathrm{EC}}=\left(\kappa_{\mathrm{B}} / \gamma_{\mathrm{LV}}\right)^{1 / 2}$. We show that suppression of capillary-induced folding or enhancement of folding a rough membrane substrate consisting of a thin PDMS film with a soot coating ${ }^{16}$ can be achieved depending on the wetting properties of the liquid. We report the development of a carbon nanoparticle (soot) coating that is superhydrophobic for water, but is wetted by allyl alcohol and so provides a system in which both non-penetrating (Cassie-Baxter) and penetrating (Wenzel) states to be studied. Allyl alcohol is chosen as a wetting liquid because it can form an azeotropic mixture with water and, therefore, its concentration remains constant during evaporation.

To create a flexible superhydrophobic substrate, we attempted a wide range of techniques (laser etching, casting against rigid substrates, etc.) to produce nano- and microstructured superhydrophobic surfaces on membranes but with little success. The approach that was eventually successful combined the production of rapeseed oil nanoparticles ${ }^{16}$ with a process initially developed to transfer a layer of carbon nanoparticles from a glass surface onto a rigid PDMS surface to produce superhydrophobicity. ${ }^{17}$ In our work, we combined these two methods using the soot covered glass transfer 
method with the rapeseed oil nanoparticles. The advantage of using rapeseed oil, rather than ethanol, in a flame synthesis approach is that it produces much thicker smoke, reduces coating times, and can produce large surface areas displaying superhydrophobicity.

In our method, rapeseed oil was placed into a ceramic bowl with a wick and left burning for 5-7 min in order for a stable flame to develop. Glass slides were then placed in the flame for between $30 \mathrm{~s}$ and $60 \mathrm{~s}$ in order to coat the entire surface of the slide with a layer of matt black rapeseed oil soot. PDMS was mixed in a 10:1 ratio and degassed in a vacuum desiccator to remove any air bubbles trapped in the mixture. After approximately $30 \mathrm{~min}$ of degassing, the PDMS was spun onto acrylic slides to the required thickness determined by the spin speed. A prebake time of 30-35 min was required at a temperature of $60^{\circ} \mathrm{C}$ for the PDMS to become sufficiently tacky for the soot to adhere to it without becoming completely engulfed by the PDMS. When the PDMS had partially cured to a tacky state, the glass slide was gently positioned, soot side down, onto the centre of the PDMS coated slides. These were then returned to a $60^{\circ} \mathrm{C}$ environment until fully cured. Once cured, the samples were allowed to cool and then the glass slides were carefully peeled from the PDMS surface leaving the soot attached to the PDMS membrane surface as a nanoparticle coating. Excess soot was removed under running water to ensure the observed superhydrophobicity was not a liquid marble effect. ${ }^{18,19}$ The thickness of the membranes $h$ were measured using a Veeco Dektak 6 M stylus profilometer determined to within $2 \mu \mathrm{m}$ for smooth layers, and to within $3 \mu \mathrm{m}$ for the soot-coated layers.

PDMS and soot-coated PDMS membranes were first characterized by measuring the static, advancing and receding contact angles with droplets of water $\left(\gamma_{\mathrm{LV}}=72.8 \mathrm{mN} \mathrm{m}^{-1}\right)$ and azeotropic mixtures of allyl alcohol $\left(\gamma_{\mathrm{LV}}=28.0 \mathrm{mN} \mathrm{m}^{-1}\right.$ and concentration of $72 \%$ ) prior to detaching them from the acrylic slides. For water, the advancing and receding angles on soot-coated PDMS were $\theta_{A}=159^{\circ} \pm 2^{\circ}$ and $\theta_{R}=153^{\circ} \pm 2^{\circ}$, respectively, compared to $\theta_{A}=116^{\circ} \pm 2^{\circ}$ and $\theta_{R}=92^{\circ} \pm 2^{\circ}$, respectively, for uncoated PDMS. For allyl alcohol, the advancing and receding angles on soot-coated PDMS were $\theta_{A}=56^{\circ} \pm 3^{\circ}$ and $\theta_{R} \sim 0^{\circ}$, respectively, compared to $\theta_{A}=64^{\circ} \pm 2^{\circ}$ and $\theta_{R}=44^{\circ} \pm 2^{\circ}$, respectively, for uncoated PDMS. Particularly relevant to the wrapping is the receding contact angle. Allyl alcohol with a near zero receding contact on soot-coated membrane is consistent with a Wenzel state in which the liquid penetrates between the surface features of the soot. For water, on the other hand, the soot is superhydrophobic with high advancing contact angle and low contact angle hysteresis so is consistent with a Cassie-Baxter in which the liquid does not penetrate into the features of the soot-coated surface.

To determine the effect of the soot coating on the rigidity of the PDMS membrane, the Young's modulus, $E$, was determined using a cantilever deflection method. In the method used, one end of a strip of PDMS was fixed whilst the other, free end, was deflected under the action of its own weight. Measurements of the maximum vertical deflections of the free end, $y_{\max }$, were carried out on PDMS membranes with widths $W=1 \mathrm{~mm}$ and varying lengths $\ell$ from 4.00 to $8.50 \mathrm{~mm}$ (to within $0.06 \mathrm{~mm}$ ). The thickness $h$ was $64 \mu \mathrm{m}$ and $82 \mu \mathrm{m}$ for uncoated and soot-coated membranes, respectively. To ensure minimal residual bending during peeling from the slides, the membranes were first floated in isopropyl alcohol (IPA) before careful removal. For each length, measurements were carried out with a given surface of the membrane facing both upwards and downwards. Deflection was independent of direction (to within 20\%) for uncoated PDMS, and $y_{\max }$ was taken to be the average value of the two measurements. However, deflections for soot-coated PDMS were smaller by a factor $\sim 2.5$ when the coated-side of membranes was facing downwards than when facing upwards.

Since uncoated PDMS is a flexible material with $E$ varying in the range $50 \mathrm{kPa}-4 \mathrm{MPa}$ ), ${ }^{20,21} y_{\max }$ is expected to exceed $\sim 20 \%$ of the beam lengths in long cantilevers. The linear small deflections equation for uniformly loaded beams which gives $y_{\max }=P \ell^{4} / 8 E I$, where $P=\rho g h$ is the weight of the membrane per unit length and unit width, $\kappa_{\mathrm{B}}=E I$ is the bending rigidity of the beam, and $\rho$ and $g$ are the density of the cantilever and acceleration due to gravity, respectively, is not applicable for $y_{\max } / \ell>0.2$. In our case $h \ll W$, and so the cantilevers can be considered as thin plates with $E I=E h^{3} / 12\left(1-\nu^{2}\right)(\nu \sim 1 / 2$ is the Poisson ratio) which in turn gives $y_{\max }=3\left(1-\nu^{2}\right) \rho g \ell^{4} / 2 E h^{2}$ for small deflections, so $E$ can be determined from $y_{\max }$ provided $\ell$ and $h$ are measured accurately. For large deflections with $y_{\max } / \ell>0.2$, we used the non-linear calibration curve relating $y_{\max } / \ell$ to $P \ell^{3} / 8 E I$ to determine $E .^{22,23}$ For uncoated membranes, we determined $E=(1.1 \pm 0.1) \mathrm{MPa}$, a value consistent with the $10 \%$ concentration of cross linker and the baking times reported by Furad et al. ${ }^{20}$ and Lopes and Bonaccurso. ${ }^{21}$ The soot coating induces a $20 \%$ increase in rigidity of PDMS to $E=(1.3 \pm 0.1) \mathrm{MPa}$ when facing upwards but a threefold increase to $E=(3.4 \pm 0.2) \mathrm{MPa}$
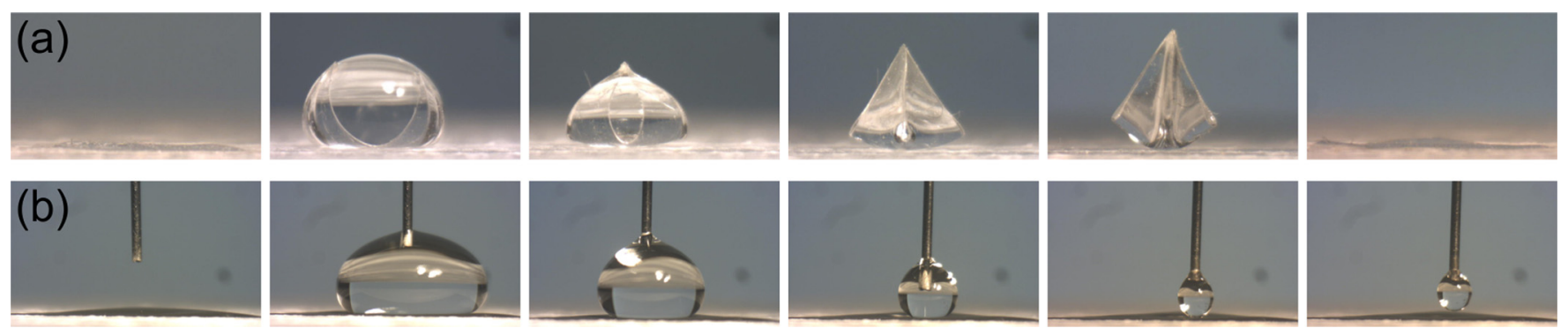

FIG. 1. (a) Image sequence showing a triangle of uncoated PDMS folding to a tetrahedral pyramid as a droplet of water evaporates. (b) Image of a droplet of water on soot-coated (superhydrophobic) PDMS membrane showing suppression of folding. 

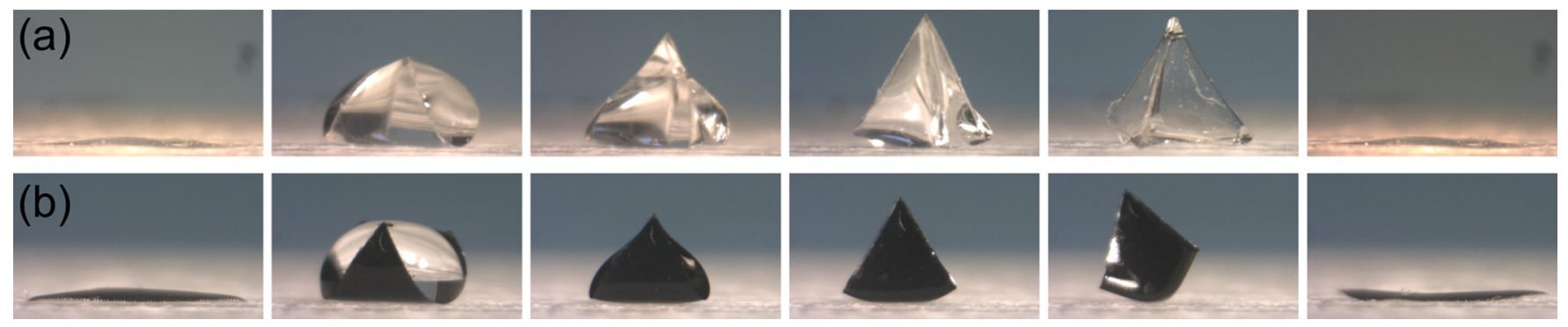

FIG. 2. Image sequence showing (a) a triangle of uncoated PDMS, and (b) soot-coated PDMS folding as a droplet of azeotropic solution of allyl alcohol evaporates.

when facing downwards. This latter value is relevant for droplet wrapping. The increased rigidity may be attributable to jamming of the nanoparticles under large inward deflections. This increase in $E$, nevertheless, leaves the coated PDMS as a flexible membrane.

To determine the critical length, $L_{\text {crit }}$, for capillaryinduced droplet wrapping, equilateral triangular shaped membranes of PDMS and soot-coated PDMS membranes varying in thickness between $35 \mu \mathrm{m}$ and $85 \mu \mathrm{m}$ were produced. For each thickness of PDMS, a range of triangle edge lengths, $L$, in steps of $0.25 \mathrm{~mm}$, were cut using a laser cutter. Each thickness was placed on a hydrophobic surface (to minimize adhesion to the supporting surface) and was observed over the entire evaporation process following the deposition of a droplet of water or an azeotropic solution of allyl alcohol of sufficient size to cover the surface of the triangle. When droplets of water were allowed to evaporate from sufficiently large uncoated PDMS membranes (Figure 1(a)) or when droplets of allyl alcohol were allowed to evaporate from uncoated or soot-coated PDMS membranes (Figures 2(a) and 2(b), respectively), they induced a wrapping transition. As the thickness of the PDMS was increased, the wrapping transition moved to larger volume droplets with lower curvature (and hence bending energy). The drying regime for each size was recorded in order to determine the critical length; here, we define this as the smallest side length for which $50 \%$ of the triangles of that size folded. However, when water drops were deposited on soot-coated (superhydrophobic) PDMS membranes, wrapping was completely suppressed for all thicknesses $h$ and for triangles of lengths up $L=15 \mathrm{~mm}$ (Figure 1(b)).

In order to make a direct comparison with the measurements of Py et al. ${ }^{6}$ for wrapping of PDMS by droplets of water, the elastocapillary length $L_{E C}$ was determined for each thickness $h$ using $L_{E C}=\sqrt{\kappa_{\mathrm{B}} / \gamma_{L V}}=\sqrt{E h^{3} / 12 \gamma_{L V}\left(1-\nu^{2}\right)}$, with a value of $E=1.1 \mathrm{MPa}$ obtained from our cantilever deflection measurements. Figure 3 shows the dependence of $L_{\text {crit }}$ on $L_{E C}$ for uncoated PDMS together with those of Py et al. for comparison. The dashed line divides triangular samples into two types: those above the line are wrapped by evaporating droplets, whilst those below are not. For all membranes and both liquids, $L_{E C} \ll L_{C}$, where $L_{C}=\sqrt{\gamma_{L V} / \rho_{L} g}$ is the capillary length $\left(\rho_{\mathrm{L}}\right.$ is the density of the liquid), so gravity effects are not expected to be important. There is a good agreement between the two sets of data with a linear fit of our data giving $L_{\text {crit }}=13.4 L_{E C}$ (to within $10 \%$ ) compared to $L_{\text {crit }}=11.9 L_{E C}$ for Py et al. This gives us confidence that our estimate of $E$ is reasonably accurate. Interestingly, both data sets can be better fitted by a non-zero intercept straight line.

In order to compare the wrapping of uncoated membranes by water and allyl alcohol, the dependence on surface tension, the wrapping condition $L_{c r i t}>L_{E C}=\sqrt{\kappa_{\mathrm{B}} / \gamma_{L V}}$ can be written in terms of energy. Wrapping occurs when the gain in surface energy $\left(\sim \gamma_{L V} L_{c r i t}^{2}\right)$ is greater than the bending energy $\kappa_{\mathrm{B}}$, i.e., when $\gamma_{L V} L_{c r i t}^{2}>\kappa_{\mathrm{B}}=E h^{3} / 12\left(1-\nu^{2}\right)$. Figure 4 shows the variation of $\gamma_{L V} L_{c r i t}^{2}$ with $\kappa_{\mathrm{B}}$ on uncoated PDMS both for water and for allyl alcohol. The dashed lines show linear fits of $\gamma_{L V} L_{c r i t}^{2}$ with $\kappa_{\mathrm{B}}$ having a slope of 152 $( \pm 10)$ for water $\left(\theta_{R}=92^{\circ}\right)$ compared to $41( \pm 4)$ for allyl alcohol $\left(\theta_{R}=44^{\circ}\right)$. The 3.7 times decrease in the slope between the two liquids indicates a dependence of $L_{\text {crit }}$ on receding contact angle; a wetting liquid with a lower receding contact angle can wrap the membrane into a tighter curve. The wrapping of soot-coated PDMS membranes in Figure 4 (with $E=3.4 \mathrm{MPa}$ ) also obeys a linear fit but with a further 1.8 times decrease in slope to $21( \pm 3)$ compared to uncoated PDMS. Allyl alcohol on soot-coated membranes $\left(\theta_{R} \sim 0^{\circ}\right)$ is a Wenzel state, and so penetrates the features of the surface, thus enhancing adhesion of the drop which, in turn, produces more powerful Capillary Origami as observed. We have thus succeeded in creating a flexible superhydrophobic coating which suppresses wrapping of a droplet of a non-wetting liquid (water) and enhances wrapping of droplet of a wetting liquid (allyl alcohol). One unexpected feature of the data, however, is the non-zero intercept value for all graphs $\sim 500 \mathrm{~nJ}$ (to within 10\%), which seems

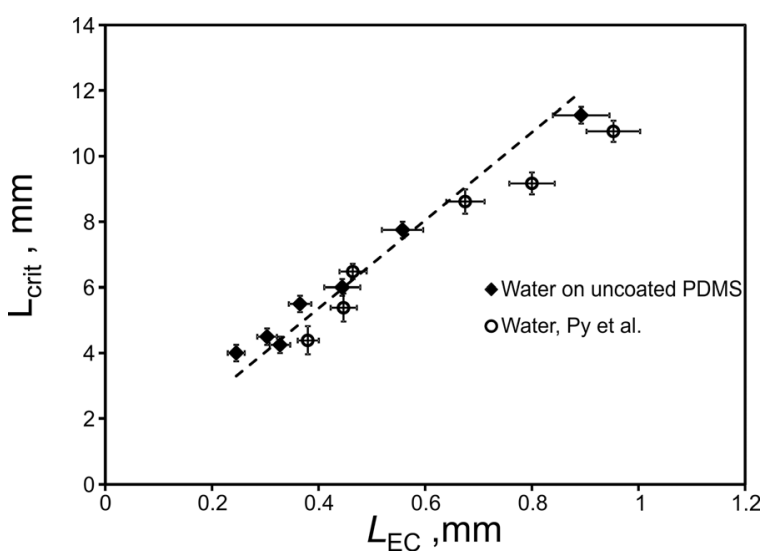

FIG. 3. The dependence of $L_{\text {crit }}$ for wrapping water droplets on uncoated PDMS on $L_{\mathrm{EC}}$ (filled diamonds). The open circles show the corresponding data of Py et al. ${ }^{6}$ for comparison. 


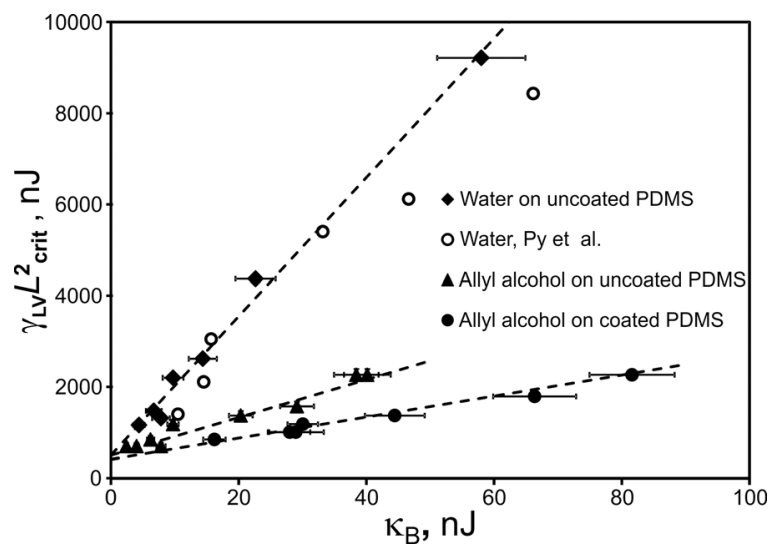

FIG. 4. The variation of $\gamma_{\mathrm{LV}} L^{2}$ crit with $\kappa_{\mathrm{B}}$ for water on uncoated PDMS (filled diamonds), and allyl alcohol on uncoated PDMS (filled triangles) and coated PDMS (filled circles). The open circles show data of Py et al. ${ }^{6}$ for comparison.

to be independent of the liquid used or surface topography and cannot be accounted for by variations in thickness of PDMS. Re-plotting the data of Py et al. gives an offset value of $670 \mathrm{~nJ}$ which is within $30 \%$ of the value obtained in this experiment. One possibility is that the experimental method using the wrapping of a triangular shaped membrane results in a dependence on the Gaussian bending rigidity.

In this work, we have shown that the contact angle can be used to control Capillary Origami and droplet wrapping. At one extreme, we have developed a method to create a flexible PDMS membrane with a superhydrophobic surface supporting a Cassie-Baxter type suspended droplet state. Water completely fails to wrap the membrane. At the other extreme, we have shown that using a wetting liquid (an azeotropic solution of allyl alcohol) enhances wrapping of the membrane by the liquid, supporting a Wenzel type penetrating droplet state. We have also shown that the critical lengths for wrapping triangular-shaped uncoated PDMS membranes are larger for water than allyl alcohol, thereby demonstrating dependence on the receding contact angle.
These measurements support the idea that wetting properties of the substrate affect its ability to wrap a droplet and that a flexible substrate can wrap more easily for smaller receding contact angles at a given surface tension of liquid and topography of surface.

This work has been supported by the U.K. Engineering \& Physical Sciences Research Council (EPSRC) under Grant EP/E063489/1. NRG is grateful to Nottingham Trent University for provision of a Ph.D. studentship.

${ }^{1}$ R. R. A. Syms and E. M. Yeatman, Electron. Lett. 29, 662 (1993).

${ }^{2}$ P. W. Green, R. R. A. Syms, and E. M. Yeatman, J. Microelectromech. Syst. 4, 170 (1995).

${ }^{3}$ R. R. Syms, J. Microelectromech. Syst. 8, 448 (1999).

${ }^{4}$ R. R. A. Syms, E. M. Yeatman, V. M. Bright, and G. M. Whitesides, J. Microelectromech. Syst. 12, 387 (2003).

${ }_{5}^{5}$ J. Bico, B. Roman, L. Moulin, and A. Boudaoud, Nature 432, 690 (2004).

${ }^{6}$ C. Py, P. Reverdy, L. Droppler, J. Bico, B. Roman, and C. N. Baroud, Phys. Rev. Lett. 98, 156103 (2007).

${ }^{7}$ C. Py, P. Reverdy, L. Doppler, J. Bico, B. Roman, and C. N. Baroud, Eur. Phys. J. Spec. Top. 166, 67 (2009).

${ }^{8}$ H. Li, X. Guo, R. G. Nuzzo, and K. J. Hsia, J. Mech. Phys. Solids 58, 2033 (2010).

${ }^{9}$ A. Takei, Y. Yoshihata, and I. Shimoyama, J. Micromech. Microeng. 21, 085009 (2011).

${ }^{10}$ S. Manakasettharn, J. A. Taylor, and T. N. Krupenkin, Appl. Phys. Lett. 99, 144102 (2011).

${ }^{11}$ S. Tawfick, A. J. Hart, and M. De Volder, Nanoscale 4, 3852 (2012).

${ }^{12}$ B. Roman and J. Bico, J. Phys: Condens. Matter 22, 493101 (2010).

${ }^{13}$ L. Gao and T. J. McCarthy, Langmuir 24, 9183 (2008).

${ }^{14} \mathrm{G}$. McHale, Langmuir 25, 7185 (2009).

${ }^{15}$ G. McHale, M. I. Newton, N. J. Shirtcliffe, and N. R. Geraldi, Beilstein J. Nanotechnol. 2, 145 (2011).

${ }^{16}$ M. Qu, J. He, and B. Cao, Appl. Surf. Sci. 257, 6 (2010).

${ }^{17}$ L. Y. Yuan, J. J. Dai, X. H. Fan, T. Song, Y. T. Tao, K. Wang, Z. Xu, J. Zhang, X. D. Bai, P. X. Lu, J. Chen, J. Zhou, and Z. L. Wang, ACS Nano 5, 4007 (2011).

${ }^{18} \mathrm{P}$. Aussillous and D. Quéré, Nature 411, 924 (2001).

${ }^{19}$ G. McHale and M. I. Newton, Soft Matter 7, 5473-5481 (2011).

${ }^{20}$ D. Fuard, T. Tzvetkova-Chevolleau, S. Decossas, P. Tracqui, and P. Schiavone, J. Microelectron. Eng. 85, 1289 (2008).

${ }^{21}$ M. C. Lopes and E. Bonaccurso, Soft Matter 8, 7875 (2012).

${ }^{22}$ J. T. Holden, Int. J. Solids Struct. 8, 1051 (1972).

${ }^{23}$ A. Ohtsuki, Appl. Mech. Mater. 24-25, 371 (2010). 